\title{
Pain Management, Coping with Stress, and Quality of Life for Women with Fibromyalgia: A Qualitative Case Study
}

\section{Euis Desy Mayangsari ${ }^{a}$, Elizabeth Kristi Poerwandari ${ }^{\mathrm{b}}$, and Mellia Christia ${ }^{\mathrm{c}}$}

$a_{\text {Faculty of Psychology, Universitas Indonesia, Depok, Indonesia; }}{ }^{b}$ Department of Clinical Psychology, Faculty of Psychology, Universitas Indonesia, Depok, Indonesia; ${ }^{c}$ Department of Clinical Psychology, Faculty of Psychology, Universitas Indonesia, Depok, Indonesia

*Corresponding author:

Elizabeth Kristi Poerwandari

Department of Clinical Psychology

Faculty of Psychology, Universitas Indonesia

Jl. Lkr. Kampus Raya, Depok, Jawa Barat Indonesia, 16424

Tel.: +62 217270004

Email address: elizabeth.kristi@ui.ac.id 


\title{
Pain Management, Coping with Stress, and Quality of Life for Women with Fibromyalgia: A Qualitative Case Study
}

\begin{abstract}
Fibromyalgia (FM) is a chronic illness characterized by pain that spreads throughout the body, accompanied by other symptoms. In general, fibromyalgia sufferers experience fatigue, mood disorders, dizziness, sleep disturbances, and difficulty in concentrating. Other symptoms are also often found in people with fibromyalgia, such as impurity, headaches, and intestinal irritation. Individuals with fibromyalgia are usually reported to have a decreased quality of life, decreased functioning status, and make greater demands on health services. This study was conducted using qualitative methods for pain management, coping with stress, and quality of life for women with fibromyalgia. In addition, this research can help provide an overview of fibromyalgia sufferers as the illness relates to psychological conditions and efforts to overcome changes that occur both physically and psychologically. The method used was in-depth interviews conducted on five women with fibromyalgia with an age range of 25-55 years.
\end{abstract}

Keywords: fibromyalgia, coping stress, pain management, quality of life

\section{Introduction}

Fibromyalgia (FM) is a chronic illness characterized by pain that spreads throughout the body, accompanied by other symptoms. In general, people with FM experience fatigue, mood disorders, dizziness, sleep disturbances, and difficulty in concentrating (Wolfe et al., 1995). Other symptoms are often found in people with FM, namely impurity, headaches, and irritation of the intestine (Gritzner, 2011). This condition usually presents difficulties for health workers or for treatment with drugs. Individuals with FM are reported to experience decreased quality of life, decreased functioning status, and make increased demands on health services (Berger et al., 2007).FM is characterized by central nervous system pain, and even though the inclusion of stimuli from the peripheral nerves also plays a role, it is the central factor (center of centization), among many occurring symptoms (Clauw, 2014). Individuals with fibromyalgia must learn to overcome various symptoms that occur and have to make significant lifestyle changes (SarziPuttini et al., 2008). This causes high stress because the etiology of illness is still unknown and unpredictable (Gritzner, 2011). So, far, the condition of individuals with FM has raised the challenge of finding effective standard care. The choice of various types of treatment allows it to be carried out by FM individuals themselves, by such means as handling psychopharmacology, physical treatment, medical treatment, and psychosocial settings. One of the researchers working in this study has also suffered from FM and felt that it was still very difficult to understand the cause of this condition. A diagnosis that asserts that the condition cannot be cured can make the researcher skeptical of medical diagnoses. Treatment uncertainty and therapeutic success have a marked effect on the psychological conditions that occur. The pain that arises knows no time limit and can occur suddenly, limiting activity. This condition makes it difficult for the researcher to fulfill social and work responsibilities. Interpersonal relationships become disrupted because the pain that emerges forces the researcher to do 
something to reduce pain, such as halting activities and avoiding contact with others. In addition, there are particular concerns for the researcher, as a woman, that this pain can bring dangerous impacts in the future, such as on the ability to have children, or mobility issues that will continue to get worse over time. Therefore, this study aims to understand the experience of people with FM. Researchers have tried to explore the flow of the illness using the theoretical framework shown in Fig. 1.

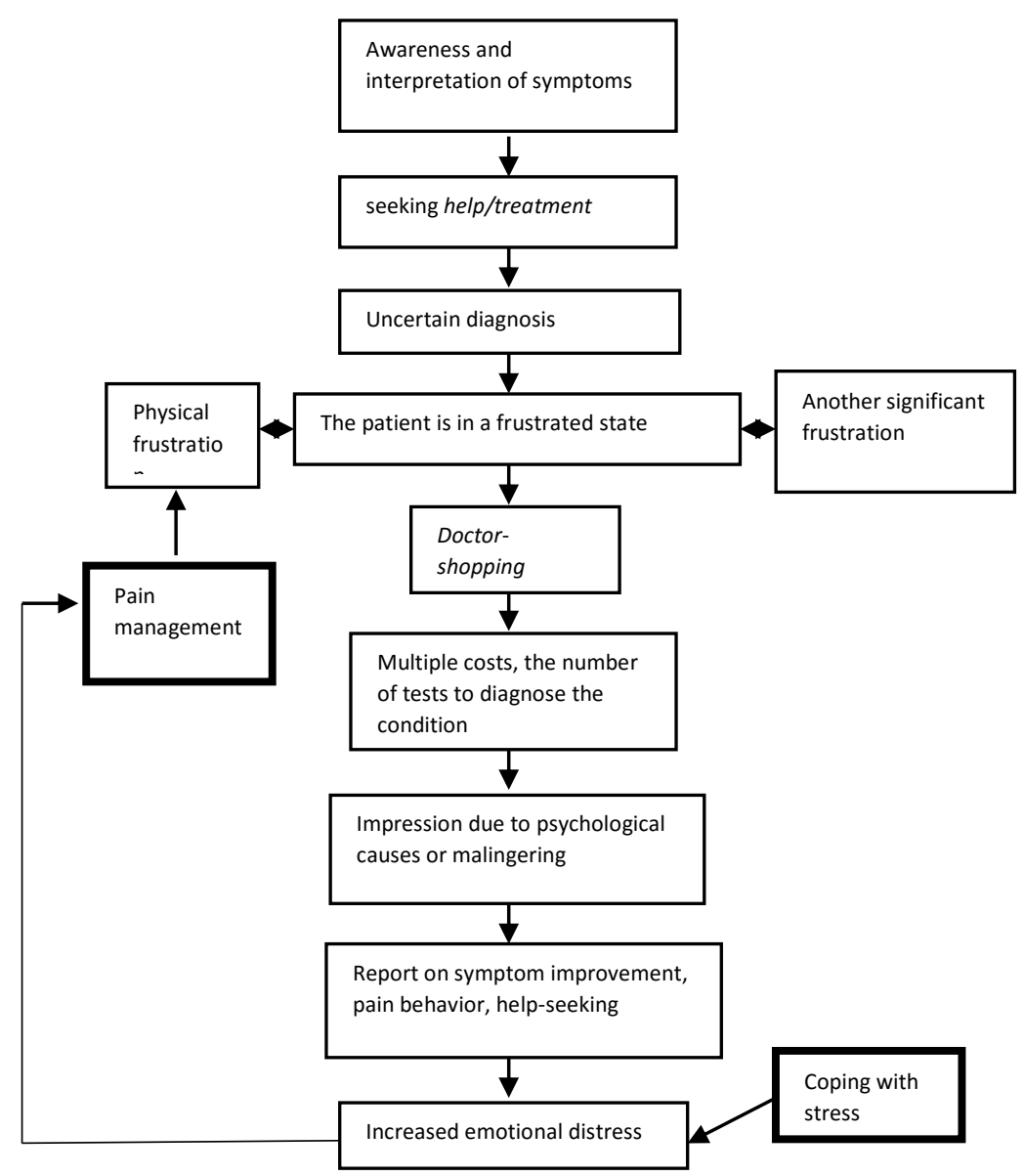

Fig.1. Adapted from Natural history of symptoms: A patients' perspective (McCarberg \& Clauw, 2009)

Currently, the concept of FM in use is as introduced by Smythe and Moldofsky in the mid1970s. The name change from Fibrositis (-itis = inflammation) is a reflection of the increasing occurrence where there is no inflammation in the connective tissue in individuals with this condition, but instead there is -algia (pain). Progress in knowledge about FM was also developed by the American College of Rheumatology (ACR), published in 1990. The classification of the criteria required to describe the condition is that individuals have a chronic widespread pain (CWP) history and identified at least 11 or more than 18 tender points at the time of examination. These ACR classification criteria are intended for research, being used to standardize the definition of FM. Unfortunately, many practitioners also use these criteria for routine clinical practice to diagnose patients individually. 
The ACR criteria for FM require that a person has a history of CWP and finding of at least 11 and up to 18 possible tender points during the examination. The tender point represents nine pairs of known body parts, usually along the musculotendinous insert. If the individual reports pain in the palpable area with a pressure of $4 \mathrm{~kg}$, this is considered a positive tender point. Approximately $25 \%$ to $50 \%$ of individuals with CWP usually have 11 or more tender points, and FM criteria are met. FM prevalence is usually quite high in rural areas or non-industrial areas as exemplified in the case of the United States.

FM is reported to be found in women more often than men, with a 9:1 ratio and is associated with rheumatological conditions. The prevalence of fibromyalgia in women in the United States is around $3.4 \%$, while for men prevalence is at $0.5 \%$, and costs due to fibromyalgia reach US\$ 9 billion per year (Rossy et al., 1999). Many clinical experts uniquely associate fibromyalgia with women who have high levels of distress, and this is based on (1) ACR criteria requiring 11 weak points. (2) Most of the findings in a fibromyalgia study coming from clinical samples from temporary care centers, where healthcare is being applied to a higher state of comorbidity in psychological conditions and psychiatry.

Stress produces many physiological system changes in the body that can affect health. A clear relationship is found between stress, body reactions, and illness. One conton is found in a study that shows high job stress levels to be associated with high blood pressure and an increase in abnormal heart pressure (Schnall et al., 1989 in Sarafino, 2004). Other studies have shown that heredity displays greater reactivity in women than in men in this regard (McAdoo et al., 1990; Ratliff-Crain \& Baum, 1990; Turner \& Hewitt, 1992 in Sarafino, 1994).

McCarberg and Clauw (2009) describe women with widespread pain, fatigue, and various symptoms that accompany it to be slow, following body sensations and pain, and experiencing cognitive attention changes that will increase the likelihood of strengthening and distorting the symptoms of pain. This condition will lead to self-acceptance as being disabled individuals. On the other hand, the emergence of fear will prevent women from having the opportunity to undertake activities that can exercise flexibility, endurance, and strength without causing pain or injury. Individuals with fibromyalgia view the condition as something that causes pain and is equated with injury. In addition, fatigue will encourage them to avoid activities that will reduce energy because of the fear of carrying out activities that they think will cause symptoms. This makes day-to-day routine become disturbed and greatly affects the quality of life.

Quality of life is defined as the individual's perception of their position in life, viewed from the cultural context and the value system in which they live and their relationship to goals, hopes, standards, and concerns (WHO, 1996). The aspect of quality of life in this study refers to the quality of life aspects of the World Health Organization Quality of Life shorter version (WHOQoL-BREF) because it covers the overall quality of life. There are six aspects of quality of life according to WHOQOL (Power in Lopers \& Synder, 2004) including physical health, psychological well-being, level of independence, social relations, relationships with the environment, and spiritual circumstances. These six aspects are narrowed down to four aspects 
of the WHOQol-BREF instrument namely: physical health, psychological well-being, social relations, and relationships with the environment.

People who suffer from fibromyalgia tend to discontinue efforts to develop new strategies for managing pain. Instead, they will switch to a passive coping strategy, such as inactivity, medication, or alcohol to reduce emotional stress and a number of symptoms that are involved. Besides that, they will also tend to free themselves from personal responsibility in managing the symptoms that arise. They will choose to depend on their family or healthcare provider (McCarberg \& Clauw, 2009).

Based on the explanation above, it can be seen that FM is closely related to the emergence of stress when dealing with symptoms. In addition, efforts to overcome pain also become an issue that will be faced by FM sufferers as an effort in treatment. Various physical experiences, such as pain and emotional distress are closely related to the quality of life of the individual. Therefore, researchers conducted research to see how coping with stress and managing pain impact on the quality of life of individuals who suffer from FM, especially women, who have a higher likelihood of getting this illness.

\section{Method}

\section{A. Purpose and Specific Aim}

This study was conducted to see how pain management can affect the quality of life of women with FM. In addition, this study can help provide an overview of FM patients related to psychological conditions, and efforts to overcome changes that occur both physically and psychologically.

\section{B. Design, Sample, and Setting}

Research on pain management, coping with stress, and the quality of life of women with FM uses a qualitative research design with a phenomenological approach. This phenomenological approach is defined as the subjective experience and awareness of one's perspective in terms of various types of subjects encountered (Moloeng, 2010). The phenomenological approach is also explained as a science that aims to describe specific phenomena or the appearance of things/events as life experiences (Speziale \& Carpenter, 2003). In this study, the life experience that researchers want to explore is the experience of women who have been diagnosed with FM, where it is believed that women with FM experience life events that are different from other, healthy women. In addition, each woman with FM will also give a different description of her experience. Therefore, in achieving the objectives of this study, researchers sought to obtain this information by emphasizing the wealth, breadth and depth of the experience of women with FM (Speziale \& Carpenter, 2003). There are four steps in the process of descriptive phenomenology, namely: bracketing, intuiting, analyzing, and describing (Polit, Beck, \& Hungler, 2001).

The selection of participants for this study uses the purposive sampling method. Besides being a sampling strategy that is commonly used in qualitative research, it is also a sample research method based on criteria relevant to the research objectives (Mack, Woodsong, MacQueen, 
Guest, \& Namey, 2005). In the study of pain management, coping with stress, and quality of life in women with FM, the criteria used in the selection of participants were that they should be women aged 20-40 years, with a diagnosis of FM and still under treatment or having physiotherapy, able to communicate well and speak Indonesian, and willing to be participants who want to provide information about pain management, coping with stress, and quality of life in women with fibromyalgia.

The selection process began by identifying prospective participants who met the criteria of the study, namely women who had been diagnosed with fibromyalgia by doctors. Once the prospective participants were identified, and in accordance with the selection criteria, the researchers introduced themselves and established a relationship of mutual trust with prospective participants, conveying the intent, purpose, and procedures related to the proposed research. Prospective participants were then asked about their willingness to become participants in this study. If the prospective participants agreed, they were asked to complete and sign a consent form as an indication of informed consent. Furthermore, once the participants had agreed to voluntarily participate in this research process, and a relationship had been established with the researcher, interviews were arranged at a time and place convenient to the participants.

\section{Data Collection}

The process of data collection through interviews was conducted at the homes of the participants at the agreed time and in accordance with the wishes of the participants. The place and time to carry out the interview process was in accordance with the agreement between participants and researchers. The timings for interviews that researchers commonly used for all participants ranged, on average, from 45 minutes to one hour. The process of collecting data in the validation process was done after the data transcript and data analysis of each participant was completed. The data analysis was then validated directly by the participants to get conformity between the statements submitted by the participants and the meanings found by researchers through data analysis. Through this process, this validation was also carried out to ensure that the transcripts and analyses compiled by the researchers were approved by participants as reflecting their actual experience.

The data collection tool in this study was the researcher himself as a direct instrument, a measuring tool for stress coping (Brief COPE), a measurement tool for search management (Folsom Pain Management), and a quality of life measurement tool (WHOQoL-BREF). Researchers play a role in extracting information as deeply as possible regarding the needs and objectives in this research, assisted by three measuring tools to complete information related to the variables to explore further. During the data collection through interviews conducted with the participants, researcher recorded all the results of the interviews using Sony Voice Recorder devices, chosen for their sound quality and recording duration. Additionally, notes were taken to record keywords and key events. 


\section{Data Explication and Analysis}

Data analysis in qualitative research is clearly different from quantitative research, in terms of dividing and combining the activities of data collection, data analysis, and writing of results. This data analysis is carried out together with data collection, data interpretation, and narrative report writing (Creswell, 2003). In addition, according to Bogdan and Biklen (1982, in Moleong 2010), data analysis is an effort to organize data, sort it into units that can be managed, synthesize, find patterns, find important and useful insights, and decide what will deliver results and be shared with others. The stages of data analysis that the researchers used in this study, are in accordance with the stages developed by Colaizzi (1978, in Speziale \& Carpenter, 2003). The reason researchers chose the data analysis stage from Coalizzi, was because the stages submitted were very easy for researchers to understand, and simple and detailed in describing the stages of the data analysis process. The stages of data analysis that researchers carry out based on the stages of Colaizzi are:

- Describe the phenomenon studied. In this case the phenomenon is FM in women and the associated pain management, coping with stress, and quality of life, and the researchers first gained an understanding of the condition in order to form the basis of this research.

- Gather descriptions of the phenomenon based on information conveyed by participants. To get an insight into the management of pain, coping with stress, and the resultant quality of life in women with FM, researchers conducted interviews with several female participants who had FM. The results of the interviews were written in the form of transcripts.

- Read all descriptions of the phenomenon that have been obtained from participants. The researcher reads the interview transcripts to get an understanding of the experience that the participants have conveyed.

- Reread the transcript, then quote significant statements. After reading transcripts of interviews, researchers carry out the process of re-reading and repetition, in order to find significant keywords consistent with research objectives.

- Decipher the meaning of each significant statement. From the keywords found that are in accordance with the purpose of the research, the researcher then re-examines each of the selected keywords to interpret their intended meaning. At this stage the researcher can form several categories of keywords.

- Organizing a collection of meanings that form into groups of themes. A collection of meanings in terms of ii is a category formed from keywords. At this stage the collection of meanings or categories that are formed is grouped again by assessing the similarity of meanings or comparing them, so that they can form themes and sub-themes.

- Write a complete and in-depth description. At this stage, researchers have described each theme that emerged as the findings related to pain management, coping with stress, and the quality of life of women with FM. The description of the results of this study is outlined in the research results.

- Return to participants to get validation from the description of the results. Researchers carried out direct validation of the results with the participants to confirm that their themes and findings were the actual experiences of women with FM. 
- Validate the results of data analysis directly with participants. When the researcher returned to the participants and discussed the findings of this study, there were several participants who gave input regarding keywords that supported a category or sub-theme, and some even provided additional information regarding the keywords in question. However, the results of the validation with participants did not change the theme that had been formed. With the additional information and clarification of the keywords found during validation, the analysis was repeated and the new data merged into the appropriate themes.

Trustworthiness in qualitative research is very important (Golafshani, 2003). Trustworthiness in qualitative research is known as credibility, neutrality or confirmability, consistency or dependability, and applicability or transferability. These terms become important criteria in assessing qualitative research (Lincoln \& Guba, 1985 in Golofshani, 2003).

\section{Results}

The interview results show that each participant had a different experience in dealing with FM, but there are also some similar complaints felt in common when pain arising from FM occurs. Several issues emerged that detail participants' experiences in dealing with FM.

\section{A. Fear and Uncertainty}

To most of the participants FM was at first a foreign term. Diseases that are considered "foreign" cause feelings of fear and lack of clarity in the participants' mind because they have never heard of similar experiences from those closest to them. One of the participants said: "Yes, it's like being slow to think, even counting, until my husband asks to use a calculator ... the husband says 'you are slow to do simple work,' then I read an article that says FM affects reduced memory. There, I also read that FM has various effects, it feels like it wants to die, it feels heart ache when treated, I fear it leads to another more serious illness."

This shows that the uncertainty of diagnosis due to foreign and diverse symptoms raises its own fears. In addition, the accompanying decline in ability makes friends and family confused and worried. This condition indirectly becomes a source of anxiety that will bring other negative feelings. Concerns about the severity of illness will also result in behavioral restrictions in daily life. This is usually done to minimize high physical activity ... "I used to be sick, Ijust wanted to go up the stairs."

\section{B. Helplessness}

Participants had a lot of treatment during the initial stage. That is because one treatment is not considered effective enough to deal with the pain that arises. This made the participants feel that it was not something "that was able to cope with the pain they felt ..." so it was right that if they were sick they were given therapy, but then they got sick again, they were told to go to therapy again but that was a bit troublesome too. The number of recommendations by medical personnel or advice from other people was felt to complicate the picture but without providing an instant effect of reducing pain. Not infrequently the pain caused participants to have limited 
mobility ... "Even if I wake up, I have to move very slowly like that." Not infrequently the body's stamina is depleted because it endures pain and limits activity ... "most of which hinder work sometimes are weak, sometimes like not being able to go to the office, sad not to be able to take care of a child during illness and not being able to wake up, it feels like no bones.".

\section{Fear of Dying}

Unbearable pain raises its own fear. Participants thought that the pain they were suffering was very serious and could cause death ... "the chest often throbbed on the left, the doctor finally decided to ECG, after that it turned out that my heart was normal, it was suspected that this was due to stress, the dontor said that I was panic attack and I was afraid to die, I told to the doctor that it feels like it's like to die, I'm always scared of dying, the doctor encourages me to get up."

\section{Surrender}

The sense of excitement that comes continuously makes the participants want to give up and have trouble finding a way out ... "what? if you are worried ya still worry, even become a conversation, but did less work feels very tired, headache, especially if you already have children, it will be more stressful, I just hope the people around me always support." Participants become accustomed to physical limitations and do not want to attempt other treatment that can cause failure and disappointment.

\section{E. Increasing Pain}

Increased pain is the symptom most often felt by people with FM. The more pain the day will increase and this will be exacerbated by physical activity. Participants are not aware that their activity is causing them to become ill because it is a normal, routine activity. When the pain comes on suddenly and is more acute than previously, it causes participants to be scared and shocked ... "yesterday suddenly it could move and wake up from sleep." The pain that came on at first was only considered normal pain arising from fatigue, but the intensity and duration were long and torturous enough to raise other suspicions ... "before I only felt the usual pain due to fatigue until the afternoon, I also sat too much but suddenly I wanted waking up but unable to move, only being able to lie down the next day, the doctor said that I had to be treated when taken home, but the doctor initially said I was tired." Participants also experienced serious symptoms. "At first I was dizzy when my mother and father were out of town, finally I went from the office to my own motorbike to the hospital to check and continued the lab check, I had to be treated, when I was treated I was treated on USG and X-ray, no infection was found but trombocite too high such as blood cancer. The doctor said I had indicated leukemia and had to be observed first. Absolutely was feel down, right." This increase in pain further decreases the patient's confidence but confidence can heal.

\section{F. Distrust}

A bad experience of therapy or feeling pain makes it difficult to tell other people, as they may not understand. Avoidance of environments and interactions with many people is considered the most effective way ... "I prefer to be alone, since 2012 I have no relationship, I feel alone, doing activities such as watching or hanging out on my own, looking for a quiet place to watch 
by myself, not too like being in crowded even in the office, if there is a gathering, I prefer myself to be busy with work."

\section{G. The Stigma Associated with FM}

Symptoms experienced by people with FM are unknown to the community. Most people think this is strange and it raises questions. The participants are sometimes reluctant to explain again or even too lazy to give information repeatedly, because the response is sometimes negative. "I used to be treated every year but like DBD, Typhus because of the worst college tiredness because I did not know the cause until I was sentenced to various diseases such as fibromyalgia at this time, the doctor just explained with various sources and literarur because this disease used to be rarely found." "If people ask, I am confused to explain, I just say muscle aches, but people do not believe it, because I have difficulty walking."

\section{H. Decreased Quality of Life}

Quality of life is one of the aspects that is felt to be subject to change, namely a decrease ... "I was not active as before, did not feel comfortable in the body. If you get tired easily now ...... 'now it's easy to complain, when the activity isn't as heavy as before."'

\section{Discussion}

The pain that occurs in participants is caused by various conditions. One of the things that made the five participants distressed was when there were pains that appeared suddenly on parts of the body. Usually the pain that arises is on a continuous basis. Unbearable pain made participants speculate that the pain felt was a symptom of certain illnesses., and this is what motivated participants to seek help or treatment. Usually the process of finding help occurs because of an acute, disabling event such as sudden paralysis or an accident. This condition can be caused by ignoring symptoms or, if in case of an accident, the symptoms appear after the body part has been seriously injured. In this treatment the participants mostly received a diagnosis that could be linked to several diseases. The number of possible diagnoses, based on their symptoms, resulted in participants having to undergo several health tests and hospital treatment for a long period of time without any certainty of diagnosis.

Another condition was when a participant had not experienced a physical accident, but was seeking treatment by going to a doctor, based on symptoms of pain that had disrupted day-today activity. Limitations in carrying out activities were the reason they needed to seek treatment. It can take a long time to arrive at a FM diagnosis, and some patients are only given a temporary diagnosis based on the most prominent symptoms appearing at the time. During treatment at the hospital or seeking treatment from the doctor, the participants experienced physical frustration in the form of pain that came on regularly. Usually the pain differed in intensity, but on each occasion there was always an increase in the level of pain. This condition is in accordance with the definition of pain itself, which is an unpleasant sensory experience related to actual tissue damage and unpleasant potential which is localized to a part of the body or often referred to as destructive where the tissue feels like being pricked, burning hot, twisting, and causing feelings of fear and nausea (Potter, 2012). 
The condition of pain is such that participants feel the urge to deal with it. This strain of pain is carried out pharmacologically and nonpharmacologically. Pharmacologically, the most common method of pain relief is taking the medication prescribed by the doctor. This is considered the most effective way to deal with the immediate symptoms. However, knowing the side effects of long-term use, participants have tended to reduce consumption over time. Sometimes participants have tried to restrict medication to occasions when the pain is truly unbearable. In addition to taking medication, participants also apply ointments, creams, or heat pads. This method is used to divert the pain with the feeling of heat caused by the ointment or pad. Physiotherapy is also used to reduce the intensity of the pain, however, the effects of physiotherapy are felt only in the short term, and would require considerable costs to be carried out for the long term.

Nonpharmacological treatment is the most frequently used because it is considered to have fewer negative side effects than medical treatment. In addition, nonpharmacological treatments are considered to have lower costs. Traditional medicine is one of the methods used, including acupuncture, massage, and energy transfer. Acupuncture and massage are considered to make a painful and tense body part more relaxed and less painful. This method is also considered easy and practical, and the costs incurred are quite affordable. However, this method does not always provide a pleasant effect. Sometimes a part of the body that is massaged will experience high levels of pain after receiving therapy. Some simple methods that can be done at home or anywhere include: lying down, sleeping, or sitting. These methods are usually the first steps taken when FM symptoms appear. This is considered to provide time for the body to adjust to the sudden onset of pain. In special cases there are participants who use extreme methods to overcome pain, such as sexual intercourse.

\section{Conclusion}

Based on the findings and discussion, it was found that the appearance of symptoms was caused by problems that caused trauma physiologically and psychologically. In the handling of pain there is no method that is truly appropriate and effective for individuals with FM. Many participants combined pharmacological and nonpharmacological treatments. In addition, coping with stress was managed with a focus on solving the problems that occur (problemfocused coping) and a focus on relieving emotions that arise due to concomitant problems (emotion-focused coping). Participants understood that action was needed to make themselves comfortable. This was done through activities that can bring excitement and entertainment, such as going out with friends or enjoying hobbies. The influence of health conditions, interpersonal relationships, work performance, and psychological effects are part of life quality. This is what some participants felt and it encouraged them to seek treatment and cope with stress. Participants felt they had difficulty in several aspects of life after experiencing FM, especially health aspects, economic aspects, aspects of interpersonal relations, and performance aspects. 


\section{References}

Berger, A., Dukes, E., Martin, S., Edelsberg, J., \& Oster, G. (2007). Characteristics and healthcare costs of patients with fibromyalgia syndrome. International Journal of Clinical Practice, 61, 1498-1508.

Clauw, D. J. (2014). Fibromyalgia: A clinical review. JAMA, 311, 1547-1555.

Creswell, J. W. (2003). Desain penelitian: Pendekatan kualitatif dan kuantitatif. Jakarta: KIK Press.

Golafshani, N. (2003). Understanding reliability and validity in qualitative research. The Qualitative Repost. 8, 597-607.

Moleong, L. J. (2010). Metode penelitian kualitatif (revision ed.). Bandung: P.T Remaja Rosdakarya.

Wolfe, F., Ross, K., Anderson, J., Rusell, I. J., \& Herbert, L. (1955). The prevalence and characteristic of fibromyalgia in the general population. Arthitis and Rheumatism. 38, 19-28. 